\title{
Morphology Time Point Reference
}

National Cancer Institute

\section{Source}

National Cancer Institute. Morphology Time Point Reference. NCI Thesaurus. Code C117634.

The point in time that acts as a fixed reference point to a morphology finding. 